\title{
Effects of acute exercise on drug craving, self-esteem, mood and affect in adults with poly-substance dependence: feasibility and preliminary findings
}

Brief Report to Drug and Alcohol Review

\author{
Maren Mikkelsen Ellingsen MPsych ${ }^{1}$, Sunniva Launes Johannesen RN ${ }^{1}$, \\ Egil W. Martinsen $\mathrm{PhD}^{1}$, \& Mats Hallgren $\mathrm{PhD}^{2 *}$
}

1. Department for Inpatient Treatment of Substance Misuse, Division of Mental Health and Addiction, Oslo University Hospital, Norway.

Email for Egil Martinsen: e.w.martinsen@medisin.uio.no

Email for Maren Mikkelsen Ellingsen: mamiel@ous-hf.no

Email for Sunniva Launes Johannsen: SUNLAU@ous-hf.no

Shared contact details for Martinsen, Mikkelsen and Johannsen: Phone +47-22923466 (no fax available) Postal address: 1039 Blindern 0315, Oslo, Norway.

2. Department of Public Health Sciences, Karolinska Institutet, Stockholm, Sweden. Postal address: Level 6, Solnavägen 1e, 171 77, Stockholm, Sweden.

Words: 1820

Running header: Exercise for substance abuse

*Correspondence: Mats Hallgren, Department of Public Health Sciences, Karolinska Institutet, level 6, Solnavägen 1e, 171 77, Sweden. Mats.hallgren@ki.se; ph. 0728735451 (no fax available) 


\title{
Effects of acute exercise on drug craving, self-esteem, mood and affect in adults with poly-substance dependence: feasibility and preliminary findings
}

\author{
Abstract \\ Background: Novel treatments for substance abuse are needed. Acute bouts of exercise can \\ improve mood states in non-clinical populations, but effects in those with poly-substance \\ dependence are understudied. We examined the feasibility and short-term effects of three types \\ of exercise on drug cravings, self-esteem, mood, and positive/negative affect in nine poly-drug \\ dependent inpatients. Methods: Using a cross-over design, changes in the four study outcomes \\ were assessed immediately before exercise and on four separate occasions post-exercise \\ (immediately after, then at one, two and four hours post-exercise) enabling patterns of change \\ over time (ANCOVA) to be observed. Results: Participants were willing and able to engage in \\ different non-laboratory based exercises. Football was associated with non-significant short-term \\ reductions in drug-cravings. A similar trend was seen for circuit-training, but not walking. \\ Football and circuit-training were associated with brief improvements in mood and \\ positive/negative affect. No adverse events were reported. Conclusion: Football, circuit training \\ and walking are feasible therapeutic activities for inpatients with poly-substance dependence. \\ Controlled trials are needed to determine the long-term effects of these activities.
}

Key words: Exercise, acute, substance abuse, physical activity, drugs 


\section{Background}

Recent studies suggest that exercise may reduce the craving for tobacco in smokers [1], and the urge to drink in those with alcohol dependence [2]. However, studies exploring the effects of acute exercise among illicit drug users remain scarce [3]. In a recent study with 24 stimulant dependent participants, drug craving reduced significantly during and 50 minutes after stationary cycling at a moderate intensity, compared to a control condition (active reading). In another study, 92 subjects were randomly assigned to mild, moderate and vigorous stationary cycling and an active reading control group [4]. Reductions in cravings were greater among subjects in the moderate and vigorous exercise groups, and the effects sustained 50 minutes after the sessions.

Despite some progress, several issues are still not addressed, including: (1) whether acute exercise performed in non-laboratory settings can benefit those with poly-substance dependence;

(2) if the effect of single exercise bouts varies across different forms of exercise; and (3) how long the effects last after the sessions have finished. We examined the short-term effects of three types of exercise in poly-drug dependent inpatients.

\section{Method}

Study design, setting and participants We conducted a feasibility study to compare the shortterm effects of three different exercises (football/soccer, circuit-training and walking), conducted in non-laboratory settings, on drug craving, mood and self-esteem. The study was conducted at the Department for Inpatient Treatment of Substance Misuse in the Division of Mental Health and Addiction at Oslo University Hospital, Norway. Most patients attending the clinic are male poly-substance users with co-morbid mental health problems; typically mood and personality disorders, aged 26 years and above. Recognizing the somatic and psychological benefits of 
exercise, the clinic offers voluntary exercise sessions to all patients. Although the facility has capacity for up to 29 patients, there were only 23 at the unit when the study was conducted. Whether or not patients receive physical activity in their treatment plan is determined by the patient (based on interest) and their treatment team (based on assessment of the patient's physical and mental health). Of the 23 patients admitted at the time, 4 were there for short term stabilization (i.e. non-voluntary treatment), and were not invited. Of the remaining 19 patients, 11 were assessed by the clinical team as suitable for group physical activity. Of these 11 patients, one did not participate because they were about to be discharged, and the other patient declined. We deliberately chose not to recruit more participants for this feasibility study, as we wanted to give as much information and support to a smaller group of suitable inpatients. We propose to extend this study with a larger randomized controlled trial in near future.

All participants had a clinician-diagnosed DSM-IV substance use disorder. The only exclusion criterion was medical contraindications to physical exercise (none met this criterion). All participants were provided with written and verbal information about the study and signed an informed consent form. Ethical approval for the study was not sought as all patients attending the clinic had already consented to voluntarily participate in exercise activities as part of their treatment. Data on participant's education, housing situation, previous drug use and habitual exercise were collected from patient files or through brief interviews.

\section{Measures}

Five self-report measures were completed on five occasions. We selected measures that can be completed quickly, and are amenable to repeated administrations. Assessments were taken 
immediately before each exercise, and on four separate occasions following exercise; immediately after, then at one, two and four hours post-exercise.

(1) Drug craving was assessed using a single-item Visual Analogue Scale (VAS) ranging from 0 (no craving for drugs) to 10 (strong craving for drugs). Participants were instructed to indicate how strong their craving for drugs felt at that moment.

(2) Changes in overall mood were assessed with the Feeling Scale [5]; a single-item Likert scale ranging from -5 (very bad) to +5 very good. Participants were instructed to indicate how they felt at that moment. The scale has been used in previous acute exercise studies [6].

(3) The Positive and Negative Affect Schedule (PANAS) [7] assessed two dimensions of affect. Participants self-rate 20 items on a 5-point Likert scale ranging from 1 (very slightly/not at all) to 5 (extremely). Half the items represent positive affect, the other half negative affect.

(4) Self-esteem was measured using the 10-item Rosenberg Self-Esteem Scale [8], which measures global self-esteem; that is, both positive and negative feelings about the self. All items are scored on a 4-point Likert scale from 1 (strongly disagree) to 4 (strongly agree). Items 2, 5, 6, 8, 9 are reverse scored. Higher scores indicate higher self-esteem.

(5) Borg's Rating of Perceived Exertion Scale (RPE) [9] was administered five minutes after each exercise session to assess how strenuous the exercises was perceived by participants. The single-item scale ranges from 6 (no exertion at all) to 20 (maximal exertion).

\section{Exercise interventions and procedure}


Participants completed three one-hour supervised group exercise sessions in the same order with a one-day 'washout' period between sessions. All exercises were undertaken on-site at the clinic between 9:30am and 11am. The following three exercise activities were tested in the order shown: (1) An outdoor football game was played outdoors on a field approximately half the size of a regular football oval. Patients played in two teams; 4 in one team, 5 in the other. Two staff members also participated - the staff nurse who organized the three exercise sessions, and a sports educator (one on each team). Scores were kept with the aim of winning, but the match was friendly. To ensure participants remained hydrated there were two short breaks lasting 3 minutes each. At the start of the match, participants were instructed to 'give their best effort'.

(2) A circuit-training session using machine weights was undertaken in an exercise training facility at the hospital ward. Participants were asked to do ten repetitions of each exercise (10 in total), consisting of upper and lower body exercises, and there was a brief (1-2 minute) transition between exercises, enabling time to recover and adjust the machine intensity. Participants were instructed to adjust the level of intensity so they could perform between 10-15 repetitions and feel moderately strained on the last few repetitions. Before the circuit-training session, all participants were given instructions on the appropriate use of the machines by a qualified instructor. The circuit included, in the following order: seated leg-press, seated chest-press, abdominal crunch, seated leg-extension, shoulder-press, inter thigh exercises, seated rowing, seated torso rotation, hyperextension and hanging leg-raises; and (3)A 45-minute group walking session was completed with a staff nurse supervising. The group walked continuously on a dirt road in a recreational area of the city at a pace that enabled participants to talk amongst each other. . All exercise sessions were supervised by a qualified nurse with additional training in sports education, and also by a second person with bachelor-level education in sports science. 
These three exercises were chosen because they include both aerobic and anaerobic training, and because they were considered suitable by the participants. All surveys were completed individually under the supervision of a staff nurse.

All patients who volunteer to exercise at the clinic are advised to remain well hydrated and to avoid caffeine and alcohol before exercise. After the final assessment, participants were informally interviewed to determine their satisfaction with the three exercise conditions and to record any adverse events.

\section{Statistical analyses}

Descriptive data (Means and SDs) were calculated for each measure. Changes over time (5 measurement points) in mood, positive and negative affect and self-esteem were assessed using repeated-measures analysis of variance (ANOVA) with Bonferroni adjusted post-hoc contrasts, with an initial significance value of $\mathrm{p}<0.05$. Following adjustment, the significance level was set at $\mathrm{p}<0.012$. We conducted simple contrasts comparing the means from measures of the different time-points using the pre-exercise level (first time-point) as a reference. For the main effect of time, effect sizes are estimated using the partial Eta squared statistic $(\eta 2)(0.01=$ small, 0.06=medium, and 0.13=large effect). Post-hoc contrasts are reported as mean differences (MD) with $95 \%$ confidence intervals (CI). As data for drug craving (VAS) were non-normally distributed (Shapiro-Wilk's test), the Friedman's non-parametric Chi-square test $(\chi 2)$ was used to assess changes over time. Post-hoc comparisons were then performed using the Wilcoxon signed-rank test for related-samples (Z-test). Two participants returned questionnaires with minimal missing data. These items were imputed using the last observation carried forward 
method and a sensitivity analysis performed. As results were nearly identical, non-imputed outcomes are shown. All data was analyzed using SPSS version-22.

\section{Results}

\section{Participant characteristics}

Participant characteristics are shown in Table 1 . All participants were male (mean age $=36.7$ years, range $=31-44)$. Upon admission, eight patients were residing in temporary housing; one had private accommodation. Two came to the clinic directly from prison where they completed their sentence. All patients were dependent on two or more substances and cannabis was used by all participants. As shown in Table 1, habitual physical activity levels varied widely. .

Insert Table 1 here

\section{Exercise effects on drug craving}

Figure 1-A: There were no main effects of time for football $\left(\chi^{2}(4)=8.17, p=0.08\right)$, circuit-training $\left(\chi^{2}(4)=7.73, p=0.12\right)$, or walking $\left(\chi^{2}(4)=8.07, p=0.08\right)$, Figure 1-A shows that, following an initial reduction, mean scores on drug craving increased in the football and circuit-training groups above baseline levels two and four hours post-exercise.

Exercise effects on mood, positive/and negative affect, and self-esteem 
Mood (Figure 1-B). There was a main effect of time for both football $(F(4,5)=6.58, p=0.001$, $\left.\eta^{2}=0.56\right)$ and circuit-training $\left(F(4,5)=5.50, p=0.003, \eta^{2}=0.49\right)$ with small effect sizes.. Mean scores improved from pre-exercise to post-exercise for circuit-training, but the change was not statistically significant: $(\mathrm{MD}=-1.57,95 \% \mathrm{CI}=-3.16,0.02, p=0.053)$.

Positive affect (Figure 1-C). There were no main effects of time for football, circuit-training or walking, however, a positive trend was observed among football participants. $(F(4,5)=3.43$, $\left.p=0.021, \eta^{2}=0.40\right)$.

Negative affect (Figure 1-D). For football, there was a significant main effect of time with a small effect size $\left(F(4,5)=6.77, p=0.01, \eta^{2}=0.53\right)$. From pre-exercise, negative affect reduced across all four time-points, but the changes were not statistically significant (post-exercise; $\mathrm{MD}=5.28,95 \% \mathrm{CI}=0.45,10.11, p=0.03),(1$-hour; $\mathrm{MD}=6.71,95 \% \mathrm{CI}=0.63,12.79, p=0.03),(2-$ hours; $\mathrm{MD}=7.42,95 \% \mathrm{CI}=1.25,13.60, p=0.02$ ), (4-hours; $\mathrm{MD}=6.14,95 \% \mathrm{CI}=0.95,11.33$, $p=0.02$ ). There were no significant main effects of circuit-training or walking.

Self-esteem (Figure 1-E). The main effect of time was not statistically significant for football, circuit-training or walking. However, mean scores changed in a positive direction from pre to post-exercise in all three groups with larger magnitude changes in the football and circuittraining groups.

\section{Perceived intensity of exercise}

Scores on Borg's RPE were as follows: football (mean=13.4, SD=2.2), 'somewhat hard'; circuittraining (mean=14.1, $\mathrm{SD}=1.7)$, 'somewhat hard'; and walking (mean=9.6, $\mathrm{SD}=1.9)$, 'very light'. 
Insert Figure 1 here

\section{Discussion}

Participants were willing and able to complete brief exercise activities, and no adverse events were reported. Given our small sample and the nature of this feasibility study, which is underpowered to detect statistically significant results, conclusions draw from the results remain tentative. Although preliminary, results suggest that exercise activities such as football, when performed in naturalistic settings, and at intensities rated as 'somewhat hard' may help to attenuate drug cravings, at least temporarily. Circuit-training could have similar effects, this should be assessedwith adequately powered controlled trials. . The 'rebound' in drug craving observed two and four hours post-exercise also warrants replication in a larger study. Speculative explanations include: (1) the timing of the assessments; exercise sessions were performed in the morning with follow-up assessments in the afternoon, when cravings may naturally rise; (2) the increase could be related to changes in hunger and/or thirst, which may increase 2-4 hours postexercise; and (3) some patients may interpret the bodily sensations associated with exercise as being similar to drug cravings. Consistent with previous work in non-clinical populations, acute exercise appeared to enhance mood and positive affect. The largest mean changes were seen for aerobic training (football), however, weight-training has been shown previously to have beneficial effects in clinical populations [10]. Future studies should assess the effects of exercise on self-esteem, which may indirectly benefit treatment adherence and recovery. Although noconclusions can be reached regarding dose-response, we speculate that higher-intensity exercises such as football, which involve fast running, could have stronger effects on drug 
craving and mood. Positive effects of football on mood could also be mediated by social interaction. Limitations include the low $n$, and non-counterbalanced design with minimal washout between sessions. . Future studies should replicate these assessments using larger participant samples and a randomized study design.

\section{References}

1. Ussher MH, Taylor AH, Faulkner GE. Exercise interventions for smoking cessation. Cochrane Database Syst Rev 2014:CD002295.

2. Ussher M, Sampuran AK, Doshi R, West R, Drummond DC. Acute effect of a brief bout of exercise on alcohol urges. Addiction 2004;99:1542-1547.

3. Wang DS, Wang YQ, Wang YY, Li RN, Zhou CL. Impact of Physical Exercise on Substance Use Disorders: A Meta-Analysis. PloS one 2014;9.

4. Wang DS, Zhou CL, Zhao M, Wu XP, Chang YK. Dose-response relationships between exercise intensity, cravings, and inhibitory control in methamphetamine dependence: An ERPs study. Drug Alcohol Depend 2016;161:331-339.

5. Hardy CJ, Rekeski WJ. Not What, But How One Feels: The Measurement of Affect During Exercise. J Sport Exerc Psychol 1989;11:304:317.

6. Rejeski WJ, Gauvin L, Hobson ML, Norris JL. Effects of baseline responses, in-task feelings, and duration of activity on exercise-induced feeling states in women. Health Psychology 1995;14:350-359. 
7. Watson D, Clark LA, Tellegen A. Development and validation of brief measures of positive and negative affect: the PANAS scales. J Pers Soc Psychol 1988;54:1063-1070.

8. Rosenberg M: Society and the adolescent self-image. Princeton, NJ: Princeton University Press, 1965.

9. Borg G, Ljunggren G, Ceci R. The Increase of Perceived Exertion, Aches and Pain in the Legs, HeartRate and Blood Lactate during Exercise on a Bicycle Ergometer. Eur J Appl Physiol O 1985;54:343-349.

10. Herring MP, O'Connor PJ, Dishman RK. The Effect of Exercise Training on Anxiety Symptoms Among Patients A Systematic Review. Arch Intern Med 2010;170:321-331. 
Figure 1 (A-E) legend

Football

...... Walking 
Author statements:

\section{Disclaimer}

The views expressed in this publication are the sole responsibility of the authors and do not necessarily reflect the views of the affiliated organizations.

\section{Acknowledgements}

Authors wish to thank the participants who volunteered for the study.

\section{Disclosure Statement}

The authors have no conflicts of interest to disclose.

\section{Funding}

None to declare.

\section{Authors Contribution}

MH and EWM wrote the first draft. MH conducted the statistical analyses. MME and SLJ implemented the study, collected participant data and performed preliminary data analyses. EWM conceived the study, supervised project implementation and writing the manuscript. All authors have read and agreed to the final version of the manuscript 